\title{
High spatial resolution and intrinsically strain-insensitive distributed temperature sensing based on stimulated Brillouin scattering in gas
}

Flavien Gyger, Fan Yang, Luc Thévenaz

Flavien Gyger, Fan Yang, Luc Thévenaz, "High spatial resolution and intrinsically strain-insensitive distributed temperature sensing based on stimulated Brillouin scattering in gas," Proc. SPIE 11199, Seventh European Workshop on Optical Fibre Sensors, 1119928 (28 August 2019); doi: $10.1117 / 12.2540683$

Event: Seventh European Workshop on Optical Fibre Sensors, 2019, Limassol, Cyprus 


\title{
High spatial resolution and intrinsically strain-insensitive distributed temperature sensing based on stimulated Brillouin scattering in gas
}

\author{
Flavien Gyger, Fan Yang and Luc Thévenaz \\ EPFL Swiss Federal Institute of Technology, Group for Fibre Optics (GFO), SCI-STI-LT \\ Station 11, CH-1015 Lausanne, Switzerland
}

\begin{abstract}
Temperature/strain cross sensitivity is a long-standing issue in Brillouin-based distributed sensors, impairing the reliability of such sensors. So far, all the proposed methods perform the discrimination by measuring two quantities showing distinct responses to temperature and strain. Distributed Raman sensing enables temperature measurement without strain sensitivity. However, due to its weak signal intensity resulting from the principle based on spontaneous scattering, the spatial resolution is typically limited to $\sim 1 \mathrm{~m}$. Here, for the first time, we use stimulated Brillouin scattering in gas-filled hollow-core photonic crystal fibers for distributed temperature sensing and we demonstrate $\sim 1 \mathrm{~cm}$ spatial resolution and $0.3^{\circ} \mathrm{C}$ temperature resolution fully free of strain cross sensitivity. Substantially higher performance is obtained thanks to the higher Brillouin gain, narrower gain linewidth and relaxed optical power restrictions when compared to solid silica single-mode fiber. This opens a new avenue in high performance distributed fiber sensing based on gas nonlinear optics.
\end{abstract}

Keywords: gas-based nonlinear optics, distributed temperature sensing, Brillouin scattering, hollow-core fiber, Brillouin optical correlation domain analysis

\section{INTRODUCTION}

Distributed optical fiber sensing has been steadily developing over the past three decades and offers the unique capability of monitoring local variations of a quantity(e.g. temperature, strain, etc) all along an optical fiber. Recently, up to two millions sensing points resolved along a single fiber have been demonstrated, ${ }^{1}$ clearly demonstrating the benefits of distributed sensing over traditional counterparts based on point sensing.

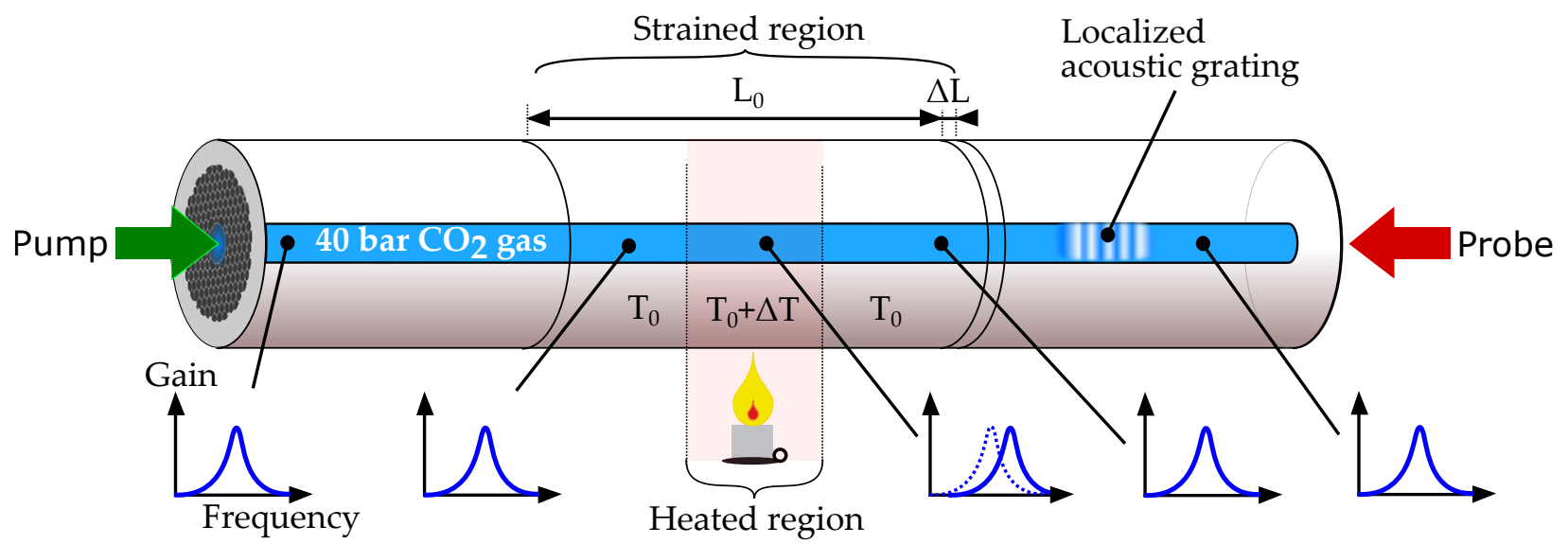

Figure 1. Distributed temperature sensing in gas-filled hollow-core photonic crystal fiber (HC-PCF) using stimulated Brillouin scattering (SBS). A localized acoustic grating is generated within the gas in the HC-PCF's core. Distributed measurement is performed by moving this grating along the whole fiber. Gas is not responsive to any strain applied to the fiber. When the temperature is changed, however, the acoustic velocity is significantly modified, leading to a frequency shift for the Brillouin gain.

Correspondence: Fan Yang, e-mail: fan.yang@epfl.ch

Seventh European Workshop on Optical Fibre Sensors, edited by Kyriacos Kalli, Gilberto Brambilla, Sinead O'Keeffe Proc. of SPIE Vol. 11199, 1119928 · C 2019 SPIE · CCC code: 0277-786X/19/\$21 · doi: 10.1117/12.2540683 
Temperature/strain cross sensitivity is currently impairing all Brillouin-based sensing systems because the acoustic velocity in a solid is indistinctly sensitive to both quantities. Many methods were proposed to solve the issue by measuring two parameters showing distinct responses to temperature and strain, such as Brillouin frequency shift (BFS) and gain value, ${ }^{2}$ BFS and birefringence in polarization maintaining fibers, ${ }^{3}$ fundamental and higher order acoustic resonances, ${ }^{4}$ combinations of Raman and Brillouin scattering ${ }^{5}$ as well as Rayleigh and Brillouin scattering. ${ }^{6}$ All these methods need to invert the response matrix to get the sensitivity of both temperature and strain, and many turn out to be ill-conditioned. One solution absent of strain cross sensitivity is based on Raman distributed sensing. ${ }^{7}$ The temperature is obtained by evaluating the power ratio between Stokes and anti-Stokes backscatterings, showing a differential cross-section resulting from the quantum thermal activation of phonons. Typical performance of such systems is $1 \mathrm{~m}$ spatial resolution at a distance of $10 \mathrm{~km}$, after a few minutes averaging. ${ }^{8}$

In this paper, we take advantage of the fluidic nature of a gaseous medium to perform strain-insensitive temperature measurement. To this end, we filled a 50 meter long HC-PCF with 40 bar $\mathrm{CO}_{2}$ and built a phasemodulated Brillouin optical correlation domain analysis (BOCDA $)^{1}$ experimental setup to generate a localized gas acoustic grating in the fiber core. Note that the loss of the HC-PCF used in this paper is $16 \mathrm{~dB} / \mathrm{km}$, which would potentially enable to cover a considerably longer sensing range (state-of-the-art loss for HC-PCF is currently $1.2 \mathrm{~dB} / \mathrm{km}^{9}$ ). Brillouin gain in gases is intrinsically much weaker than in silica under ambient conditions, but raises quadratically with pressure and turns larger than silica for pressures exceeding 20 bar. At 40 bar, the $\mathrm{CO}_{2}$ Brillouin gain is six times larger and has a linewidth seven times narrower than in standard single mode fiber (SMF), thus significantly increasing the sensor response. The grating position is scanned along the whole fiber and the local Brillouin gain spectrum is measured at each position. Any strain applied on the fiber does not impact on the gas pressure nor on the optical effective refractive index (since the tiny volume change can be fully neglected) and thus doesn't affect the Brillouin gain. On the other hand, a change in temperature significantly influences the acoustic velocity and shifts the Brillouin frequency, ${ }^{10}$ as depicted in Fig. 1. Conditions for distributed temperature measurements turn out to be better than in a solid core fiber. Indeed, the absence of gas convection in the microscopic fiber core as well as the low $\mathrm{CO}_{2}$ thermal conductivity $(0.02$ $\mathrm{Wm}^{-1} \mathrm{~K}^{-1}$ compared to $0.8 \mathrm{Wm}^{-1} \mathrm{~K}^{-1}$ for silica) impede heat propagation and create favorable conditions for high resolution distributed temperature sensing.

\section{EXPERIMENTS}

Our experimental setup essentially consists of a phase-modulated BOCDA experiment ${ }^{11}$ operated at telecommunication wavelength $(1550 \mathrm{~nm})$ and connected to the sensing system illustrated in Figure 2. For a fair comparison, identical lengths of HC-PCF and solid core SMF (ITU G.652) are jointly placed on a test bench consisting of a 4 $\mathrm{cm}$ Peltier element positioned in the middle of a $15 \mathrm{~cm}$ variable strain stage. This enables us to simultaneously apply strain and temperature over the same segment and identically for the two fiber types. Additionally the small Peltier element size is used to validate the system's high spatial resolution.

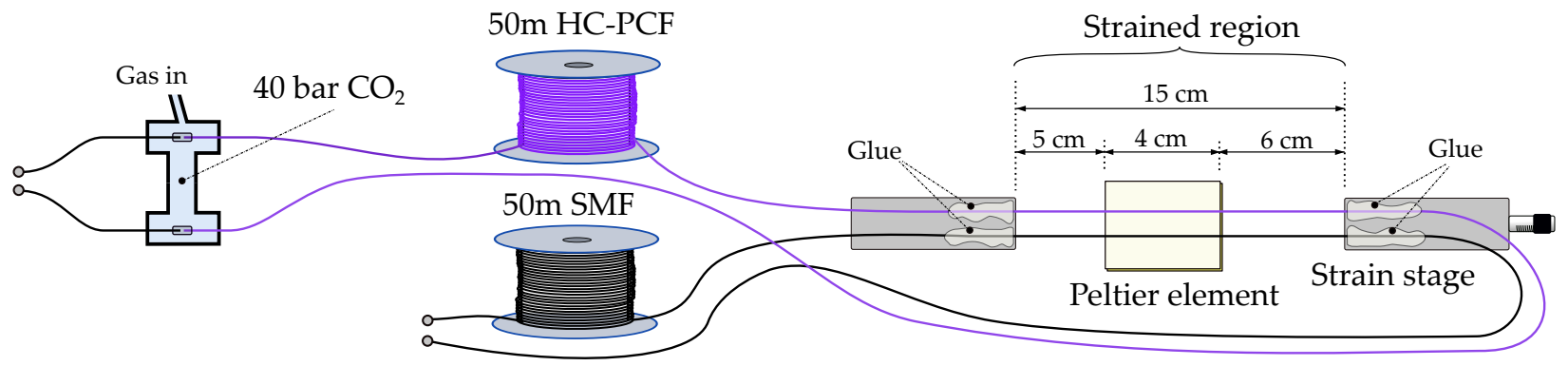

Figure 2. Sensing system. From left to right: Gas and light coupling to HC-PCF, sensing fibers, test bench. 

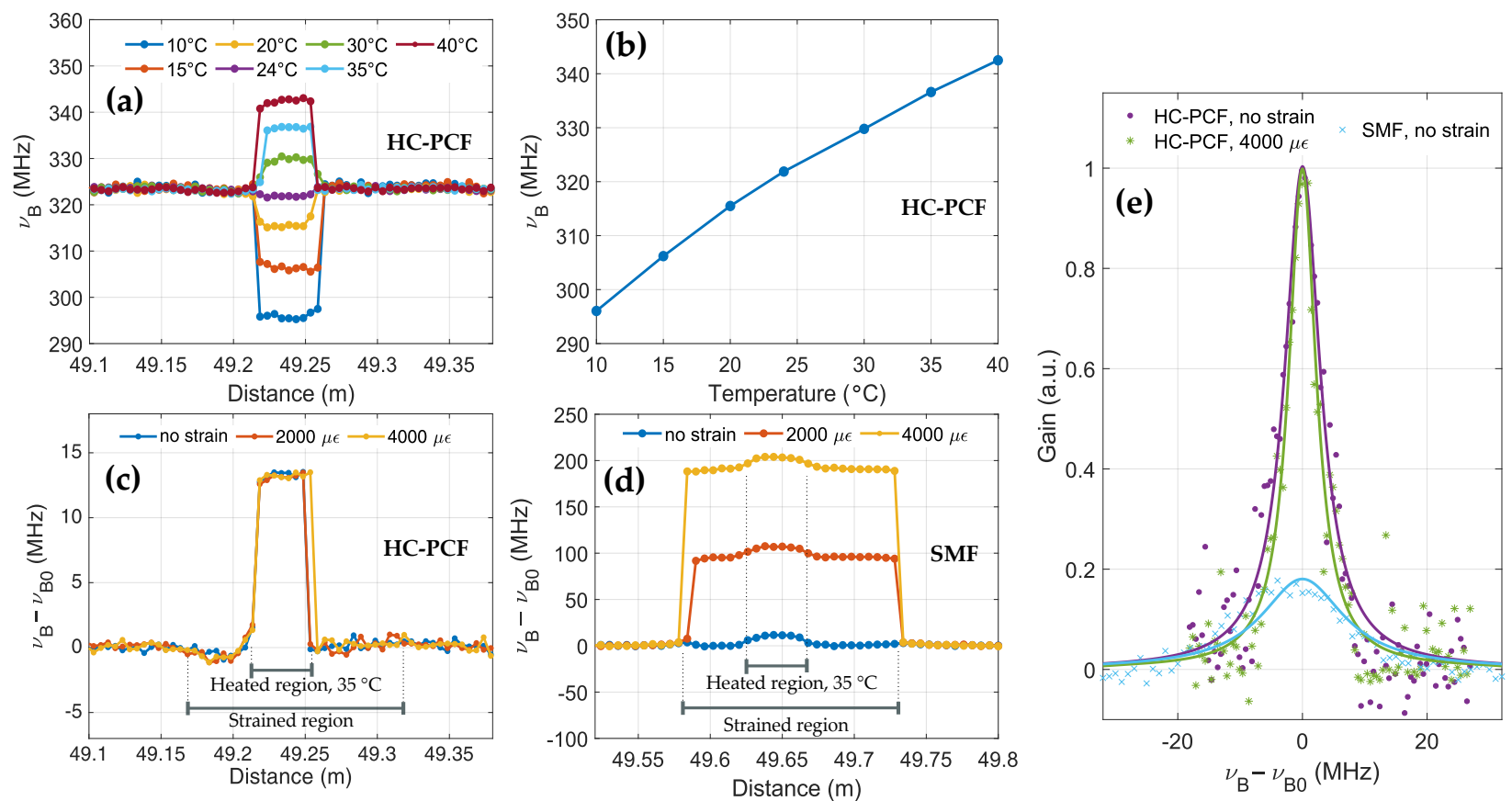

Figure 3. Experimental results. (a) HC-PCF BFS across the test bench for various preset temperatures. (b) HC-PCF $\mathrm{BFS}$ in function of the preset temperature, showing a quasi-linear curve with slope $\sim 1.2 \mathrm{MHz} /{ }^{\circ} \mathrm{C}$. (c) $\mathrm{HC}-\mathrm{PCF} \mathrm{BFS}$ shift across the test bench with temperature set at $35^{\circ} \mathrm{C}$ and different strains. (d) SMF BFS shift across the test bench with temperature set at $35^{\circ} \mathrm{C}$ and different strains. (e) Gain spectra of: HC-PCF with no strain, HC-PCF with $4000 \mu \epsilon$ and SMF, including Lorentzian fittings. $\nu_{B 0}$ is the BFS of the fiber in ambient conditions.

\section{RESULTS}

A compilation of the results is presented in Figure 3. The spatial resolution, computed as the inverse of the bit duration, were $1.28 \mathrm{~cm}$ and $2.32 \mathrm{~cm}$ for HC-PCF and SMF respectively. Measurement spectra have been acquired using a $7.8 \mathrm{~Hz}$ equivalent noise bandwidth and their peak gain frequency were estimated using a quadratic fitting. The repeatability for the HC-PCF and the SMF is experimentally estimated to be $0.3^{\circ} \mathrm{C}$ and $0.4{ }^{\circ} \mathrm{C}$ respectively. Figure 3(a) shows the HC-PCF BFS longitudinal distribution for various preset temperatures. The slightly different positioning of the abrupt transitions is due to the uncertainty in the central frequency determination when two Brillouin gain spectra overlap in presence of noise. The average HC-PCF BFS along the Peltier element as a function of the preset temperature is shown in Fig. 3(b). It must be pointed out that the response is not perfectly linear but has an average slope of $1.2 \mathrm{MHz} /{ }^{\circ} \mathrm{C}$, which is a little bit larger than for silica. Figures $3(\mathrm{c})$ and (d) compare the two fibers response at a preset temperature of $35^{\circ} \mathrm{C}$ and different applied strains: $0 \mu \epsilon, 2000$ $\mu \epsilon$ and $4000 \mu \epsilon$. As expected, a strong strain dependence is observed for the SMF, but no change is visible for the HC-PCF, validating the absence of cross sensitivity which was subsequently tested up to $1 \%$ elongation. In addition, the effect of higher spatial resolution for the HC-PCF is made visible by the sharper transition at the borders of the heated region. Figure 3(e) shows the measured gain spectra of the HC-PCF with no strain and with $4000 \mu \epsilon$, as well as the spectrum of the SMF for comparison. It can be seen that the strain did not alter the gain spectrum of the HC-PCF. The same normalization has been applied to the three spectra, highlighting the narrower linewidth as well as the higher peak gain of HC-PCF compared with SMF.

\section{CONCLUSION}

Exploiting SBS in gas to perform distributed measurements is reported for the first time and offers crucial advantages with respect to the classical approach in solid-core fibers: 
- The total absence of strain sensitivity, eliminating the recurrent issue of strain/temperature cross sensitivity when pure temperature measurements have to be performed.

- The substantially higher Brillouin gain associated to a considerably lower Brillouin frequency shift (typically 0.1 to $1 \mathrm{GHz}$ ), a narrower linewidth and an equivalent temperature sensitivity. The system can operate with no microwave elements but it requires filling the fiber with a high-pressure gas. This is however of minor technical complexity and can be addressed by splicing the hollow-core fiber to a solid-core fiber, thereby hermetically and permanently sealing the gas. ${ }^{12}$

- The use of hollow-core fibers may be a crucial asset in some extreme environments (e.g. ionizing radiations).

- The system can be designed for operation at any wavelength from vacuum ultraviolet to mid-infrared. ${ }^{13}$

- Finally, higher pump powers can be used given the reduced optical non-linearities. ${ }^{14}$

\section{ACKNOWLEDGMENTS}

The authors acknowledge the support from the Swiss National Science Foundation under grants No. 159897 and 178895, as well as their colleague Simon Zaslawski for providing the quadratic fitting algorithm.

\section{REFERENCES}

1. A. Denisov, M. A. Soto, and L. Thévenaz, "Going beyond 1000000 resolved points in a brillouin distributed fiber sensor: theoretical analysis and experimental demonstration," Light: Science 8 Applications 5(5), p. e16074, 2016.

2. T. R. Parker, M. Farhadiroushan, V. A. Handerek, and A. J. Roger, "A fully distributed simultaneous strain and temperature sensor using spontaneous brillouin backscatter," IEEE Photonics Technology Letters 9, pp. 979-981, July 1997.

3. W. Zou, Z. He, and K. Hotate, "Complete discrimination of strain and temperature using Brillouin frequency shift and birefringence in a polarization-maintaining fiber," Optics Express 17, pp. 1248-1255, Feb. 2009.

4. X. Liu and X. Bao, "Brillouin Spectrum in LEAF and Simultaneous Temperature and Strain Measurement," Journal of Lightwave Technology 30, pp. 1053-1059, Apr. 2012.

5. M. Taki, A. Signorini, C. J. Oton, T. Nannipieri, and F. D. Pasquale, "Hybrid Raman/Brillouin-opticaltime-domain-analysis-distributed optical fiber sensors based on cyclic pulse coding," Optics Letters 38, pp. 4162-4165, Oct. 2013.

6. P. C. Wait and T. P. Newson, "Landau Placzek ratio applied to distributed fibre sensing," Optics Communications 122, pp. 141-146, Jan. 1996.

7. J. P. Dakin, D. J. Pratt, G. W. Bibby, and J. N. Ross, "Distributed optical fibre Raman temperature sensor using a semiconductor light source and detector," Electronics Letters 21, pp. 569-570, June 1985.

8. G. Bolognini and A. Hartog, "Raman-based fibre sensors: Trends and applications," Optical Fiber Technology 19, pp. 678-688, Dec. 2013.

9. P. J. Roberts, F. Couny, H. Sabert, B. J. Mangan, D. P. Williams, L. Farr, M. W. Mason, A. Tomlinson, T. A. Birks, J. C. Knight, and P. S. J. Russell, "Ultimate low loss of hollow-core photonic crystal fibres," Optics Express 13, pp. 236-244, Jan. 2005.

10. A. Estrada-Alexanders and J. Trusler, "Speed of sound in carbon dioxide at temperatures between (220 and 450) K and pressures up to $14 \mathrm{MPa}$," The Journal of Chemical Thermodynamics 30(12), pp. 1589-1601, 1998.

11. A. Zadok, Y. Antman, N. Primerov, A. Denisov, J. Sancho, and L. Thévenaz, "Random-access distributed fiber sensing," Laser 83 Photonics Reviews 6(5), pp. L1-L5, 2012.

12. F. Benabid, F. Couny, J. C. Knight, T. A. Birks, and P. S. J. Russell, "Compact, stable and efficient all-fibre gas cells using hollow-core photonic crystal fibres," Nature 434, p. 488, Mar. 2005.

13. J. C. Knight, "Anti-Resonant Hollow Core Fibers," in Optical Fiber Communication Conference (OFC) 2019 (2019), paper Th3E.4, p. Th3E.4, Optical Society of America, Mar. 2019.

14. M. Azhar, G. K. L. Wong, W. Chang, N. Y. Joly, and P. S. J. Russell, "Raman-free nonlinear optical effects in high pressure gas-filled hollow core PCF," Optics Express 21, pp. 4405-4410, Feb. 2013. 